\title{
Simultaneous Invariants of Strain and Rotation Rate Tensors and Their Admitted Region
}

\author{
Igor Vigdorovich $^{1}$ and Holger Foysi ${ }^{2}$ \\ ${ }^{1}$ Institute of Mechanics, Lomonosov Moscow State University, Michurinsky Avenue 1, Moscow 119192, Russia \\ ${ }^{2}$ Chair of Fluid Mechanics, Universität Siegen, Paul-Bonatz-Straße 9-11, 57068 Siegen, Germany \\ Correspondence should be addressed to Igor Vigdorovich; vigdorovich@imec.msu.ru
}

Received 11 August 2015; Accepted 29 September 2015

Academic Editor: Christian Engstrom

Copyright (c) 2015 I. Vigdorovich and H. Foysi. This is an open access article distributed under the Creative Commons Attribution License, which permits unrestricted use, distribution, and reproduction in any medium, provided the original work is properly cited.

The purpose of this paper is to establish the admitted region for five simultaneous, functionally independent invariants of the strain rate tensor $\mathbf{S}$ and rotation rate tensor $\Omega$ and calculate some simultaneous invariants of these tensors which are encountered in the theory of constitutive relations for turbulent flows. Such a problem, as far as we know, has not yet been considered, though it is obviously an integral part of any problem in which scalar functions of the tensors $\boldsymbol{S}$ and $\boldsymbol{\Omega}$ are studied. The theory provided inside this paper is the building block for a derivation of new algebraic constitutive relations for three-dimensional turbulent flows in the form of expansions of the Reynolds-stress tensor in a tensorial basis formed by the tensors $\mathbf{S}$ and $\boldsymbol{\Omega}$, in which the scalar coefficients depend on simultaneous invariants of these tensors.

\section{Introduction}

The strain rate tensor $\mathbf{S}$ and the rotation rate tensors $\boldsymbol{\Omega}$ are the symmetric and antisymmetric parts of the velocity gradient tensor, respectively. In what follows, we will consider incompressible flow, when the tensor $\mathbf{S}$ is a deviator by virtue of the continuity equation that satisfies the extra condition $\operatorname{tr} S=0$. form

After transforming to principal axes, the tensor $\mathbf{S}$ has the

$$
\mathbf{S}=\left[\begin{array}{ccc}
\sigma_{1} & 0 & 0 \\
0 & \sigma_{2} & 0 \\
0 & 0 & \sigma_{3}
\end{array}\right] .
$$

The tensor $\boldsymbol{\Omega}$, in an arbitrary coordinate system, is written as

$$
\boldsymbol{\Omega}=\left[\begin{array}{ccc}
0 & -\omega_{3} & \omega_{2} \\
\omega_{3} & 0 & -\omega_{1} \\
-\omega_{2} & \omega_{1} & 0
\end{array}\right],
$$

where $\omega_{1}, \omega_{2}$, and $\omega_{3}$ are the vorticity vector components. If tensor (2) is written in the coordinate system connected with the principal axes of the tensor $\mathbf{S}$, the five quantities

$$
\sigma_{1}, \sigma_{2}, \omega_{1}, \omega_{2}, \omega_{3}
$$

constitute a full set of simultaneous, functionally independent invariants of the tensors $\mathbf{S}$ and $\boldsymbol{\Omega}$ [1].

If a scalar physical quantity depends on the tensors $\mathbf{S}$ and $\boldsymbol{\Omega}$, it is a function of quantities (3). For example, the theory of algebraic constitutive relations (algebraic stress models) for turbulent flows [2-5] deals with functional relations between the Reynolds-stress tensor and strain and rotation rate tensors that are calculated from the mean velocity field. Such relations are equivalent to the representations of the stress tensor in terms of expansions in a tensorial basis formed by the tensors $\mathbf{S}$ and $\boldsymbol{\Omega}$. The scalar coefficients in the expansions depend on simultaneous invariants of these tensors.

The simultaneous invariants are conveniently chosen to be quantities that, unlike (3), are easily calculable in an arbitrary coordinate system. For example, they can be the 
following five quantities that are calculated as the trace of some products of the tensors $\mathbf{S}$ and $\boldsymbol{\Omega}$ :

$$
\begin{aligned}
& \eta=\operatorname{tr} \mathbf{S}^{2}=\sigma_{1}^{2}+\sigma_{2}^{2}+\sigma_{3}^{2}, \\
& \zeta=\operatorname{tr} \mathbf{S}^{3}=\sigma_{1}^{3}+\sigma_{2}^{3}+\sigma_{3}^{3}, \\
& \xi_{n}=\operatorname{tr} \mathbf{S}^{n} \mathbf{\Omega}^{2}-\frac{1}{2} \operatorname{tr} \mathbf{S}^{n} \operatorname{tr} \mathbf{\Omega}^{2}=\sigma_{1}^{n} \omega_{1}^{2}+\sigma_{2}^{n} \omega_{2}^{2}+\sigma_{3}^{n} \omega_{3}^{2}, \\
& \quad n=0,1,2 .
\end{aligned}
$$

It is just invariants (4) and their algebraic combinations that are used in the algebraic stress models for turbulent flows (see, e.g., [2-11]). As opposed to invariants (3), which can take arbitrary, independent values, quantities (4) vary in a certain region.

The aim of the present paper is to determine the admitted region for invariants (4). Such a problem, as far as we know, has not yet been considered, though it is obviously an integral part of any problem in which scalar functions of the tensors $\mathbf{S}$ and $\boldsymbol{\Omega}$ are studied. We will calculate in passing some invariants of the tensors $\mathbf{S}$ and $\boldsymbol{\Omega}$ that are encountered in the theory of constitutive relations for turbulent flows as functions of invariants (4). In the present study, the tensor $\mathbf{S}$ is an arbitrary symmetric, traceless tensor and $\boldsymbol{\Omega}$ is an arbitrary antisymmetric tensor.

In Section 2, we determine the admitted region for the invariants $\eta$ and $\zeta$ of the strain rate tensor and calculate in terms of $\eta$ and $\zeta$ the invariant $\Gamma=\left(\sigma_{1}-\sigma_{2}\right)\left(\sigma_{2}-\right.$ $\left.\sigma_{3}\right)\left(\sigma_{3}-\sigma_{1}\right)$, which is needed in what follows. In Section 3 , we calculate the important simultaneous invariant of the tensors $\mathbf{S}$ and $\boldsymbol{\Omega} \Lambda=\Gamma \omega_{1} \omega_{2} \omega_{3}$, which in a sense is independent of invariants (4), since it can be determined in terms of them only with ambiguity in sign. In Section 4 , on the basis of the representation for the invariant $\Lambda$, the admitted region for invariants (4) is determined.

To calculate new invariants in terms of quantities (4), two basic relationships are used: the Cayley-Hamilton identity and finite isotropic relations between two tensors, which exist, if the tensors have a diagonal form in one coordinate system [1].

\section{Invariants of the Tensor $S$}

For the traceless tensor $\mathbf{S}$, the Cayley-Hamilton identity [1] reads

$$
\mathbf{S}^{3}=\frac{1}{2} \eta \mathbf{S}+|\mathbf{S}| \mathbf{I},
$$

where I is the identity matrix. After calculating the trace of both sides of equality (5), we get

$$
|\mathbf{S}|=\sigma_{1} \sigma_{2} \sigma_{3}=\frac{1}{3} \zeta .
$$

To this equality, we add two more

$$
\begin{aligned}
\sigma_{1}+\sigma_{2}+\sigma_{3} & =0 \\
\sigma_{1} \sigma_{2}+\sigma_{2} \sigma_{3}+\sigma_{3} \sigma_{1} & =-\frac{1}{2} \eta .
\end{aligned}
$$

The latter is obtained after squaring equality (7) and making use of the definition of the invariant $\eta$. Equations (6)-(8) imply that the eigenvalues $\sigma_{i}$ owing to the Viete theorem are the roots of the cubic equation

$$
\sigma^{3}-\frac{1}{2} \eta \sigma-\frac{1}{3} \zeta=0
$$

and therefore have real values under the condition

$$
\sqrt{6}|\zeta| \leqslant \eta^{3 / 2}
$$

which specify the admitted region for two invariants of the strain rate tensor with zero trace.

To calculate the invariant

$$
\Gamma=\left(\sigma_{1}-\sigma_{2}\right)\left(\sigma_{2}-\sigma_{3}\right)\left(\sigma_{3}-\sigma_{1}\right),
$$

we consider the matrix

$$
\Sigma=\left[\begin{array}{ccc}
\sigma_{2}-\sigma_{3} & 0 & 0 \\
0 & \sigma_{3}-\sigma_{1} & 0 \\
0 & 0 & \sigma_{1}-\sigma_{2}
\end{array}\right] .
$$

Since $\boldsymbol{\Sigma}$ and $\mathbf{S}$ (1) have a diagonal form in the same coordinate system, they should be related by an isotropic relation [1]

$$
\Sigma=\delta_{0} \mathbf{I}+\delta_{1} \mathbf{S}+\delta_{2} \mathbf{S}^{2},
$$

where $\delta_{0}, \delta_{1}$, and $\delta_{2}$ are scalar coefficients. By calculating the trace of both sides of equality (13) and then multiplying it consecutively by $\mathbf{S}$ and $\mathbf{S}^{2}$ and taking the trace, we obtain the system of equations

$$
\begin{aligned}
\Delta\left[\begin{array}{l}
\delta_{0} \\
\delta_{1} \\
\delta_{2}
\end{array}\right] & =-\left[\begin{array}{l}
0 \\
0 \\
\Gamma
\end{array}\right], \\
\Delta & =\left[\begin{array}{lll}
3 & 0 & \eta \\
0 & \eta & \zeta \\
\eta & \zeta & \frac{1}{2} \eta^{2}
\end{array}\right] .
\end{aligned}
$$

Here, we used the equalities

$$
\begin{gathered}
\operatorname{tr} \Sigma=0 \\
\operatorname{tr} \Sigma S=0 \\
\operatorname{tr} \Sigma S^{2}=-\Gamma
\end{gathered}
$$

and $\operatorname{tr} \mathbf{S}^{4}=(1 / 2) \eta^{2}$, the latter of which is obtained by multiplying the Cayley-Hamilton identity (5) by $\mathbf{S}$ and taking the trace.

The calculation of the inverse matrix gives

$$
\Delta^{-1}=\left(\eta^{3}-6 \zeta^{2}\right)^{-1}\left[\begin{array}{ccc}
\eta^{3}-2 \zeta^{2} & 2 \eta \zeta & -2 \eta^{2} \\
2 \eta \zeta & \eta^{2} & -6 \zeta \\
-2 \eta^{2} & -6 \zeta & 6 \eta
\end{array}\right]
$$


which enables us to determine the coefficients $\delta_{0}, \delta_{1}$, and $\delta_{2}$ and rewrite equality (13) in the form

$$
\left(\eta^{3}-6 \zeta^{2}\right) \mathbf{\Sigma}=2 \Gamma\left(\eta^{2} \mathbf{I}+3 \zeta \mathbf{S}-3 \eta \mathbf{S}^{2}\right)
$$

Multiplying (17) by $\Sigma$, taking the trace, and using again equalities (15) as well as the equality $\operatorname{tr} \Sigma^{2}=3 \eta$, which follows from (8), we obtain the representation in question:

$$
2 \Gamma^{2}=\eta^{3}-6 \zeta^{2}
$$

This formula also implies condition (10).

\section{Invariant $\Lambda$}

In what follows, we will need the invariants

$$
\begin{aligned}
& \xi_{3}=\operatorname{tr} \mathbf{S}^{3} \boldsymbol{\Omega}^{2}-\frac{1}{2} \operatorname{tr} \mathbf{S}^{3} \operatorname{tr} \boldsymbol{\Omega}^{2}=\sigma_{1}^{3} \omega_{1}^{2}+\sigma_{2}^{3} \omega_{2}^{2}+\sigma_{3}^{3} \omega_{3}^{2}, \\
& \xi_{4}=\operatorname{tr} \mathbf{S}^{4} \boldsymbol{\Omega}^{2}-\frac{1}{2} \operatorname{tr} \mathbf{S}^{4} \operatorname{tr} \boldsymbol{\Omega}^{2}=\sigma_{1}^{4} \omega_{1}^{2}+\sigma_{2}^{4} \omega_{2}^{2}+\sigma_{3}^{4} \omega_{3}^{2},
\end{aligned}
$$

which are formed by analogy to invariants (4). Multiplying the Cayley-Hamilton identity (5) consecutively by $\Omega^{2}$ and $\mathbf{S} \boldsymbol{\Omega}^{2}$, taking the trace, and using equalities (4), we get

$$
\begin{aligned}
\operatorname{tr} S^{3} \Omega^{2} & =\frac{1}{2} \eta \xi_{1}-\frac{2}{3} \zeta \xi_{0}, \\
\operatorname{tr} S^{4} \Omega^{2} & =\frac{1}{2} \eta \xi_{2}+\frac{1}{3} \zeta \xi_{1}-\frac{1}{2} \eta^{2} \xi_{0} .
\end{aligned}
$$

Now the representation of invariants (19) in terms of invariants (4) can be written in the form of the matrix equality

$$
\begin{aligned}
& {\left[\begin{array}{l}
\xi_{0} \\
\xi_{1} \\
\xi_{2} \\
\xi_{3} \\
\xi_{4}
\end{array}\right]=\mathbf{W} \xi} \\
& \mathbf{W}=\left[\begin{array}{ccc}
1 & 0 & 0 \\
0 & 1 & 0 \\
0 & 0 & 1 \\
\frac{1}{3} \zeta & \frac{1}{2} \eta & 0 \\
0 & \frac{1}{3} \zeta & \frac{1}{2} \eta
\end{array}\right], \\
& \boldsymbol{\xi}=\left[\begin{array}{l}
\xi_{0} \\
\xi_{1} \\
\xi_{2}
\end{array}\right] .
\end{aligned}
$$

Consider the invariant

$$
\Lambda=-\operatorname{tr} \mathbf{S}^{2} \Omega^{2} \mathbf{S},
$$

the direct calculation of which gives $\Lambda=\Gamma \omega_{1} \omega_{2} \omega_{3}$.
The matrixes

$$
\boldsymbol{\Theta}=\left[\begin{array}{ccc}
\omega_{1}^{2} & 0 & 0 \\
0 & \omega_{2}^{2} & 0 \\
0 & 0 & \omega_{3}^{2}
\end{array}\right]
$$

and $\mathbf{S}$ should be also related by the isotropic relation

$$
\Theta=\alpha_{0} \mathbf{I}+\alpha_{1} \mathbf{S}+\alpha_{2} \mathbf{S}^{2}
$$

Calculating the trace of both sides of equality (24) and then multiplying this equality consecutively by $\mathbf{S}$ and $\mathbf{S}^{2}$, and taking the trace, we obtain the system of equations

$$
\Delta\left[\begin{array}{l}
\alpha_{0} \\
\alpha_{1} \\
\alpha_{2}
\end{array}\right]=\xi
$$

with the same matrix (14) on the left-hand side. The coefficients in question are specified by the equality

$$
\left[\begin{array}{l}
\alpha_{0} \\
\alpha_{1} \\
\alpha_{2}
\end{array}\right]=\Delta^{-1} \xi
$$

where the matrix $\Delta^{-1}$ has the form (16).

Multiplying equality (24) by the matrix

$$
\boldsymbol{\Pi}=\left[\begin{array}{ccc}
\omega_{2}^{2} \omega_{3}^{2} & 0 & 0 \\
0 & \omega_{3}^{2} \omega_{1}^{2} & 0 \\
0 & 0 & \omega_{1}^{2} \omega_{2}^{2}
\end{array}\right]
$$

and calculating the trace yield

$$
\begin{aligned}
3\left(\omega_{1} \omega_{2} \omega_{3}\right)^{2} & =\alpha_{n} \beta_{n}, \\
\beta_{n} & =\sigma_{1}^{n} \omega_{2}^{2} \omega_{3}^{2}+\sigma_{2}^{n} \omega_{3}^{2} \omega_{1}^{2}+\sigma_{3}^{n} \omega_{1}^{2} \omega_{2}^{2}, \\
& n=0,1,2 .
\end{aligned}
$$

Since the coefficients $\alpha_{n}$ are specified by equality (26), the invariant $\Lambda$ can be determined in terms of the invariants $\beta_{n}$.

Direct calculations give

$$
\begin{aligned}
\operatorname{tr} \boldsymbol{\Omega}^{4} & =4 \beta_{0}+2 \pi_{0}, \\
\operatorname{tr} \mathbf{S} \boldsymbol{\Omega}^{4} & =\beta_{1}-\pi_{1}, \\
\operatorname{tr} \mathbf{S}^{2} \boldsymbol{\Omega}^{4} & =\beta_{2}-\pi_{2}+\eta\left(\beta_{0}+\pi_{0}\right), \\
\pi_{n} & =\sigma_{1}^{n} \omega_{1}^{4}+\sigma_{2}^{n} \omega_{2}^{4}+\sigma_{3}^{n} \omega_{3}^{4} .
\end{aligned}
$$

On the other hand, the left-hand sides of equalities (30) can be obtained on the basis of the Cayley-Hamilton identity for the tensor $\Omega$, which reads $\Omega^{3}=-\xi_{0} \Omega$. By multiplying 
it consecutively by $\boldsymbol{\Omega}, \mathbf{S} \boldsymbol{\Omega}$, and $\mathbf{S}^{2} \boldsymbol{\Omega}$, taking the trace, and making use of (4), we get

$$
\begin{aligned}
\operatorname{tr} \boldsymbol{\Omega}^{4} & =2 \xi_{0}^{2}, \\
\operatorname{tr} \mathbf{S} \boldsymbol{\Omega}^{4} & =-\xi_{0} \xi_{1}, \\
\operatorname{tr} \boldsymbol{S}^{2} \boldsymbol{\Omega}^{4} & =\eta \xi_{0}^{2}-\xi_{0} \xi_{2} .
\end{aligned}
$$

Equations (30) and (32) imply

$$
\begin{aligned}
\alpha_{n} \beta_{n}= & \xi_{0}\left(\frac{1}{2} \alpha_{0} \xi_{0}+\frac{1}{2} \eta \alpha_{2} \xi_{0}-\alpha_{1} \xi_{1}-\alpha_{2} \xi_{2}\right) \\
& -\frac{1}{2} \pi_{0}\left(3 \alpha_{0}+\eta \alpha_{2}\right)+\alpha_{n} \pi_{n} \\
= & \xi_{0}\left[\frac{1}{2} \xi_{0}\left(3 \alpha_{0}+\eta \alpha_{2}\right)-\alpha_{n} \xi_{n}\right] \\
& -\frac{1}{2} \pi_{0}\left(3 \alpha_{0}+\eta \alpha_{2}\right)+\alpha_{n} \pi_{n} \\
= & \frac{1}{2} \xi_{0}\left[\xi_{0}^{2}-2 \alpha_{n} \xi_{n}-\pi_{0}\right]+\alpha_{n} \pi_{n} .
\end{aligned}
$$

Here, we take into account that the first equation of system (25) reads $3 \alpha_{0}+\eta \alpha_{2}=\xi_{0}$.

Now, let us calculate the quantities $\pi_{n}$ specified by (31). Multiplying (24) by $\Theta S^{n}, n=0,1,2$, calculating the trace, and taking into account equalities (4) and (19) and the fact that $\pi_{n}=\operatorname{tr} \Theta^{2} \mathbf{S}^{n}$, we have

$$
\pi_{n}=\alpha_{m} \xi_{m+n}, \quad m, n=0,1,2 .
$$

Hence, in particular, we get the equality $\pi_{0}=\alpha_{n} \xi_{n}$, which reduces (33) to the form

$$
\alpha_{n} \beta_{n}=\frac{1}{2} \xi_{0}\left[\xi_{0}^{2}-3 \pi_{0}\right]+\alpha_{n} \pi_{n} .
$$

The quantity $\pi_{0}$ is the quadratic form of the matrix $\Delta^{-1}$ :

$$
\begin{aligned}
\pi_{0} & =\frac{1}{2} \Gamma^{-2}\left[\left(\eta^{3}-2 \zeta^{2}\right) \xi_{0}^{2}+\eta^{2} \xi_{1}^{2}+6 \eta \xi_{2}^{2}+4 \eta \zeta \xi_{0} \xi_{1}\right. \\
& \left.-4 \eta^{2} \xi_{0} \xi_{2}-12 \zeta \xi_{1} \xi_{2}\right] .
\end{aligned}
$$

Equalities (21) and (34) imply

$$
\alpha_{n} \pi_{n}=\alpha_{n} \alpha_{m} W_{n+m, l} \xi_{l},
$$

where all the indices take the values 0,1 , and 2 . Hence, taking the values of the entries of the matrix $\mathbf{W}$ (21) into account, we get

$$
\begin{aligned}
\alpha_{n} \pi_{n}= & \alpha_{0}^{2} \xi_{0}+2 \alpha_{0} \alpha_{1} \xi_{1}+\left(2 \alpha_{0} \alpha_{2}+\alpha_{1}^{2}\right) \xi_{2} \\
& +2 \alpha_{1} \alpha_{2}\left(\frac{1}{3} \zeta \xi_{0}+\frac{1}{2} \eta \xi_{1}\right) \\
& +\alpha_{2}^{2}\left(\frac{1}{3} \zeta \xi_{1}+\frac{1}{2} \eta \xi_{2}\right) .
\end{aligned}
$$

By substituting into (35) equalities (36) and (38) and the values of $\alpha_{n}$ that follow from (16) and (26), we finally obtain

$$
\begin{aligned}
(6 \Lambda)^{2}= & -4 \zeta^{2} \xi_{0}^{3}-12 \zeta \xi_{1}^{3}-36 \xi_{2}^{3}+36 \zeta \xi_{0} \xi_{1} \xi_{2} \\
& -9 \eta^{2} \xi_{0} \xi_{1}^{2}+18 \eta \xi_{1}^{2} \xi_{2}+18 \eta \xi_{2}^{2} \xi_{0}-12 \eta \zeta \xi_{0}^{2} \xi_{1} .
\end{aligned}
$$

It is easy to make sure that (39) coincides with the expression that was given in [12] without deduction. As is seen in (39), the invariant $\Lambda$ can be determined in terms of invariants (4) but with ambiguity in sign.

\section{Admitted Region for the Invariants}

Equality (39) enables us to establish the admitted region for invariants (4). Polynomial (39) is homogenies in the variables $\xi_{0}, \xi_{1}$, and $\xi_{2}$. By equating it to zero, we obtain an equation which specifies a conic surface in the space $\left(\xi_{0}, \xi_{1}, \xi_{2}\right)$ with the vertex at the origin. In the variables

$$
\begin{aligned}
& s=\frac{\xi_{1}}{\xi_{0} \sqrt{6 \eta}}, \\
& t=\frac{\xi_{2}}{\eta \xi_{0}}, \\
& \varkappa=\frac{\zeta \sqrt{6}}{\eta^{3 / 2}},
\end{aligned}
$$

(39) takes the form

$$
\begin{aligned}
& 27 \xi_{0}^{-3}\left(\omega_{1} \omega_{2} \omega_{3}\right)^{2}\left(\varkappa^{2}-1\right) \\
& =54 t^{3}-27 t^{2}-54\left(3 s^{2}+\varkappa s\right) t+108 \varkappa s^{3}+81 s^{2} \\
& \quad+18 \varkappa s+\varkappa^{2} .
\end{aligned}
$$

Here we used equality (18). The parameter $\varkappa$ according to condition (10) varies over interval $[-1,1]$.

In accordance with (4), the vectors $\xi$ specified in (21) and $\boldsymbol{\mu}=\left[\omega_{1}^{2}, \omega_{2}^{2}, \omega_{3}^{2}\right]$ are related by the one-to-one relation

$$
\begin{aligned}
\boldsymbol{\xi} & =\mathbf{G} \boldsymbol{\mu}, \\
\mathbf{G} & =\left[\begin{array}{lll}
1 & 1 & 1 \\
\sigma_{1} & \sigma_{2} & \sigma_{3} \\
\sigma_{1}^{2} & \sigma_{2}^{2} & \sigma_{3}^{2}
\end{array}\right] .
\end{aligned}
$$

This follows from the fact that, in the general case, the determinant $|\mathbf{G}|=\Gamma$ is unequal to zero (it vanishes in the only case $|\varkappa|=1$ ). The invariant $\omega_{1} \omega_{2} \omega_{3}$ and therefore polynomial (41) vanish, if at least one component of the vector $\boldsymbol{\mu}$ vanishes. In that case, equality (42) is a parametric representation of a plane in the space $\left(\xi_{0}, \xi_{1}, \xi_{2}\right)$. In other words, if $\omega_{1} \omega_{2} \omega_{3}=0$, then three quantities $\xi_{0}, \xi_{1}$, and $\xi_{2}$ should be related by a linear equation of the form

$$
t=a s+\frac{1}{3} b
$$


where $a$ and $b$ are constant coefficients, which specifies a straight line in the plane $(s, t)$ and a plane in the space $\left(\xi_{0}, \xi_{1}, \xi_{2}\right)$. After substituting (43) into (41), we obtain a third-order polynomial in the variable $s$. The polynomial's coefficients should be equated to zero, which gives the four equations for quantities $a$ and $b$ :

$$
\begin{aligned}
a^{3}-3 a+2 \varkappa & =0, \\
2 b^{3}-3 b^{2}+\varkappa^{2} & =0, \\
a b^{2}-a b-\varkappa b+\varkappa & =0, \\
2 a^{2} b-a^{2}-2 \varkappa a-2 b+3 & =0 .
\end{aligned}
$$

Owing to the Viete trigonometric formula, the cubic equation (44) has three real roots:

$$
\begin{aligned}
a_{1,2} & =-2 \cos \left(\phi \pm \frac{2}{3} \pi\right), \\
a_{3} & =-2 \cos \phi, \\
\phi & =\frac{1}{3} \cos ^{-1} \varkappa .
\end{aligned}
$$

The comparison of (44) with (9) leads to

$$
\sigma_{i}=-\frac{a_{i} \sqrt{6 \eta}}{6} .
$$

It is easy to see that if $a$ is a root of (44), the other three equations (45) are satisfied at $b=\varkappa / a$. Thus, polynomial (41) can be factorized as follows:

$$
\begin{gathered}
\frac{\left(\omega_{1} \omega_{2} \omega_{3}\right)^{2}\left(\varkappa^{2}-1\right)}{2 \xi_{0}^{3}}=\left(t-a_{1} s-\frac{\varkappa}{3 a_{1}}\right) \\
\cdot\left(t-a_{2} s-\frac{\varkappa}{3 a_{2}}\right)\left(t-a_{3} s-\frac{\varkappa}{3 a_{3}}\right) .
\end{gathered}
$$

The straight lines (43) have three points of intersection in the plane $(s, t)$ with the coordinates

$$
\left(-\frac{a_{i}}{6}, \frac{a_{i}^{2}}{6}\right) \text {. }
$$

As was said above, on the straight lines (43) in the general case, one component of the vorticity vector vanishes. In points (49), two components are equal to zero. One can make sure of it by turning again to equality (42), which, when the vector $\boldsymbol{\mu}$ has only one nonzero component, is a parametric equation of a straight line that issues out of the origin in the space $\left(\xi_{0}, \xi_{1}, \xi_{2}\right)$. Such a line corresponds to a point in the plane $(s, t)$. Hence, when the vorticity vector is directed along one of the strain-rate-tensor principal axes, the invariants $\xi_{0}, \xi_{1}, \xi_{2}$ on the basis of (40) and (49) must be related by the equalities

$$
\begin{aligned}
& \xi_{1}=-\frac{a \sqrt{6 \eta}}{6} \xi_{0}, \\
& \xi_{2}=\frac{a^{2} \eta}{6} \xi_{0},
\end{aligned}
$$

in which $a$ is a root of (44). These relations again imply equalities (47).

After returning to variables (40) and taking into account equalities (47) and the definitions of invariants (4), we rewrite the first factor on the right-hand side of (48) in the form

$$
\begin{aligned}
& \left(t-a_{1} s-\frac{\varkappa}{3 a_{1}}\right)=\frac{1}{6 \xi_{0}}\left(\xi_{2}+a_{1} \xi_{1}+a_{2} a_{3} \xi_{0}\right) \\
& =\frac{1}{6 \xi_{0}}\left[\left(2 a_{1}^{2}+a_{2} a_{3}\right) \omega_{1}^{2}+\left(a_{2}^{2}+a_{1} a_{2}+a_{2} a_{3}\right) \omega_{2}^{2}\right. \\
& \left.+\left(a_{3}^{2}+a_{1} a_{3}+a_{2} a_{3}\right) \omega_{2}^{2}\right]=\frac{\omega_{1}^{2}\left(a_{1}^{2}-1\right)}{2 \xi_{0}} .
\end{aligned}
$$

Here, we used the properties of the roots of (44), which follows from the Viete theorem, particularly, the equality $a_{1}+a_{2}+a_{3}=0$. We get similarly

$$
\begin{aligned}
& \left(t-a_{2} s-\frac{\varkappa}{3 a_{2}}\right)=\frac{\omega_{2}^{2}\left(a_{2}^{2}-1\right)}{2 \xi_{0}}, \\
& \left(t-a_{3} s-\frac{\varkappa}{3 a_{3}}\right)=\frac{\omega_{3}^{2}\left(a_{3}^{2}-1\right)}{2 \xi_{0}} .
\end{aligned}
$$

Now, taking the range of variation of roots (46) into account,

$$
\begin{gathered}
1 \leqslant a_{1} \leqslant 2, \\
-1 \leqslant a_{2} \leqslant 1, \\
-2 \leqslant a_{3} \leqslant-1,
\end{gathered}
$$

we may deduce that the first and third factors on the righthand side of (48) are always nonnegative while the second one is less than or equal to zero, and the admitted region for the invariants $\xi_{0}, \xi_{1}$, and $\xi_{2}$ in the plane $(s, t)$ is a triangle with apexes in the points (49). In Figure 1(a), these triangle regions are depicted for a range of $\varkappa$ over the interval $[0,1]$. Since polynomial (41) is invariant under simultaneous change in sign of the quantities $s$ and $\varkappa$, it is sufficient to consider the case $x \geqslant 0$. The regions that correspond to positive and negative values of $\varkappa$ are symmetric with respect to the axis $t$. In the limiting case $\varkappa=1$, the triangle degenerates into a straight-line segment.

Consider the limiting case $\varkappa \rightarrow 1$ in detail. Let us introduce new variables

$$
\begin{aligned}
& \varepsilon \tau=t-a_{1} s-\frac{\varkappa}{3 a_{1}}, \\
& \sigma=t-a_{3} s-\frac{\varkappa}{3 a_{3}}, \\
& \varepsilon=\sqrt{1-\varkappa^{2}}
\end{aligned}
$$



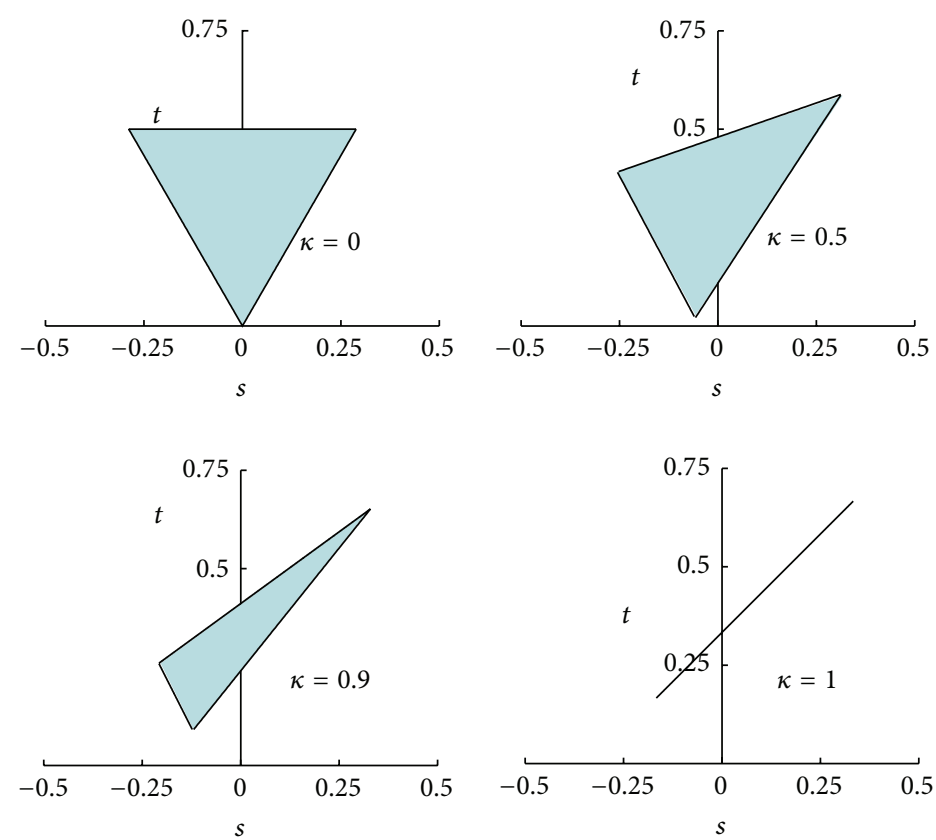

(a)
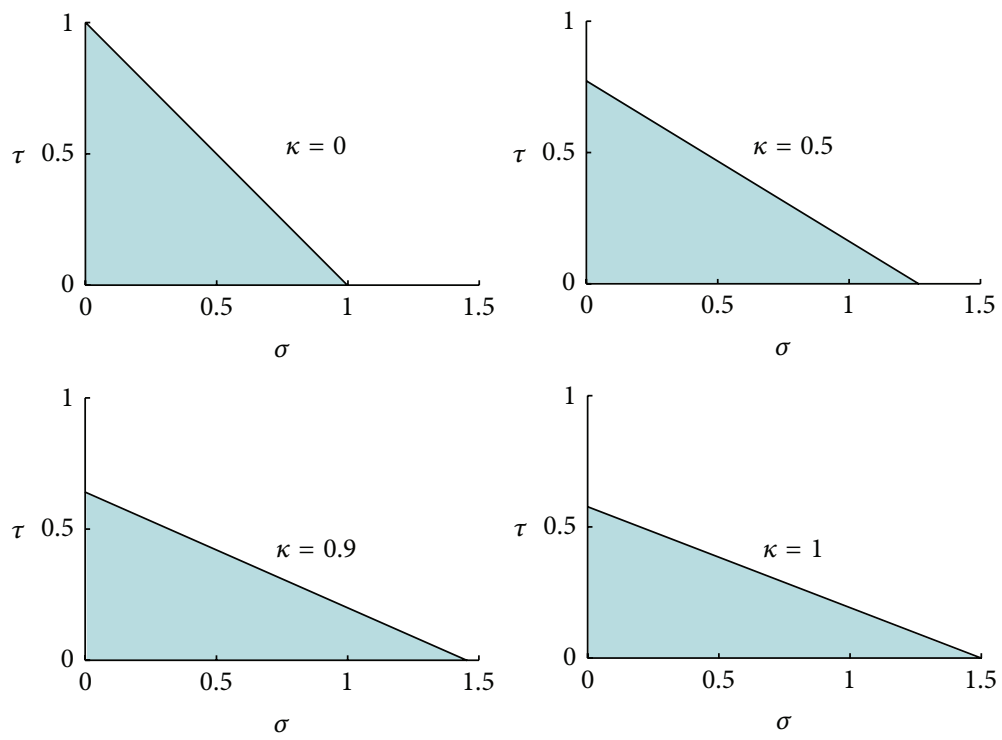

(b)

FIGURE 1: Admitted regions for the invariants $\xi_{0}, \xi_{1}$, and $\xi_{2}$ in the planes (a) $(s, t)$ and (b) $(\sigma, \tau)$ for various values of $\varkappa$.

according to which the triangle's two sides shown in Figure 1 (a) become coordinate lines of different families. By solving these equations for $s$ and $t$, we obtain

$$
\begin{aligned}
& s=\frac{\sigma-\varepsilon \tau}{a_{1}-a_{3}}-\frac{a_{2}}{6}, \\
& t=\frac{a_{1} \sigma-a_{3} \varepsilon \tau}{a_{1}-a_{3}}+\frac{a_{2}^{2}}{6} .
\end{aligned}
$$

After substituting (55) into equality (48), the latter reads

$$
\frac{\left(\omega_{1} \omega_{2} \omega_{3}\right)^{2}}{2 \xi_{0}^{3}}
$$

$$
=-\tau \sigma\left[\frac{\left(a_{2}-a_{3}\right) \varepsilon \tau+\left(a_{1}-a_{2}\right) \sigma}{\left(a_{1}-a_{3}\right) \varepsilon}+\frac{a_{2}^{3}-\varkappa}{3 a_{2} \varepsilon}\right] .
$$

Now, taking into account the asymptotic behavior of the roots $a_{i}$

$$
\begin{aligned}
& a_{1,2}=1 \pm \frac{\varepsilon \sqrt{3}}{3}+O\left(\varepsilon^{2}\right), \\
& a_{3}=-2+O\left(\varepsilon^{2}\right),
\end{aligned}
$$

$$
\varkappa \longrightarrow 1
$$


we can construct the admitted regions for the invariants in the plane $(\sigma, \tau)$, which have the form of right triangles, shown in Figure 1(b).

\section{Conclusions}

The problem of determining a functional relation between any scalar physical quantity and the strain rate and rotation rate tensors is reduced to determining a function of simultaneous scalar invariants of the tensors $\boldsymbol{S}$ and $\boldsymbol{\Omega}$, which from reasons of the ease of computation can be chosen as the quantities $\eta, \zeta, \xi_{0}, \xi_{1}$, and $\xi_{2}$. Hence, the problem of investigating the admitted region for these five invariants naturally arises. It has been established that this region can be constructed in three-dimensional space of the parameters

$$
\begin{aligned}
& s=\frac{\xi_{1}}{\xi_{0} \sqrt{6 \eta}}, \\
& t=\frac{\xi_{2}}{\eta \xi_{0}}, \\
& \varkappa=\frac{\zeta \sqrt{6}}{\eta^{3 / 2}},
\end{aligned}
$$

where it is situated between the planes $\varkappa= \pm 1$, while its boundary in each cross section $\varkappa=$ const is a triangle in the plane $(s, t)$. When the parameter $\varkappa$ tends to its limiting values \pm 1 , the triangle's area vanishes while the triangle degenerates into a straight-line segment.

In the sequel, we plan to derive new algebraic constitutive relations (explicit algebraic stress models) for threedimensional turbulent flows in the form of expansions of the Reynolds-stress tensor in a tensorial basis formed by the tensors $\boldsymbol{S}$ and $\boldsymbol{\Omega}$, in which the scalar coefficients depend on simultaneous invariants of these tensors. The result obtained in the present study will be used, in particular, to investigate the asymptotic behavior of the scalar coefficients at the boundary of the admitted region for the invariants.

\section{Conflict of Interests}

The authors declare that there is no conflict of interests regarding the publication of this paper.

\section{Acknowledgment}

This work was partially supported by the visiting grant of the Deutsche Forschungsgemeinschaft (German Research Foundation) Project no. DFG FO 674/7-1.

\section{References}

[1] L. I. Sedov, Introduction to the Mechanics of a Continuous Medium, Addison-Wesley Publishing Co, New York, NY, USA, 1965.

[2] S. B. Pope, Turbulent Flows, Cambridge University Press, Cambridge, UK, 2000.
[3] D. B. Taulbee, "An improved algebraic Reynolds stress model and corresponding nonlinear stress model," Physics of Fluids A, vol. 4, no. 11, pp. 2555-2561, 1992.

[4] T. B. Gatski and C. G. Speziale, "On explicit algebraic stress models for complex turbulent flows," Journal of Fluid Mechanics, vol. 254, pp. 59-78, 1993.

[5] D. B. Taulbee, J. R. Sonnenmeier, and K. M. Wall, "Stress relation for three-dimensional turbulent flows," Physics of Fluids, vol. 6, no. 3, pp. 1399-1401, 1994.

[6] S. S. Girimaji, "Fully explicit and self-consistent algebraic Reynolds stress model," Theoretical and Computational Fluid Dynamics, vol. 8, no. 6, pp. 387-402, 1996.

[7] S. S. Girimaji, "A Galilean invariant explicit algebraic reynolds stress model for turbulent curved flows," Physics of Fluids, vol. 9, no. 4, pp. 1067-1077, 1997.

[8] T. Jongen and T. B. Gatski, "General explicit algebraic stress relations and best approximation for three-dimensional flows," International Journal of Engineering Science, vol. 36, no. 7-8, pp. 739-763, 1998.

[9] S. Wallin and A. V. Johansson, "An explicit algebraic Reynolds stress model for incompressible and compressible turbulent flows," Journal of Fluid Mechanics, vol. 403, pp. 89-132, 2000.

[10] D. Violeau, "Explicit algebraic Reynolds stresses and scalar fluxes for density-stratified shear flows," Physics of Fluids, vol. 21, no. 3, Article ID 035103, 2009.

[11] W. M. J. Lazeroms, G. Brethouwer, S. Wallin, and A. V. Johansson, "An explicit algebraic Reynolds-stress and scalarflux model for stably stratified flows," Journal of Fluid Mechanics, vol. 723, pp. 91-125, 2013.

[12] T. S. Lund and E. A. Novikov, Parameterization of Subgrid-Scale Stress by the Velocity Gradient Tensor, Annual Research Briefs, Center for Turbulence Research, 1992. 


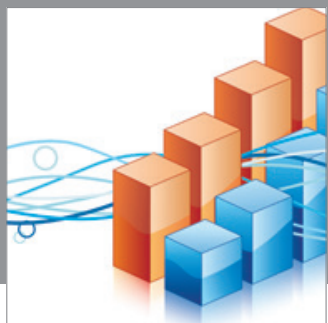

Advances in

Operations Research

mansans

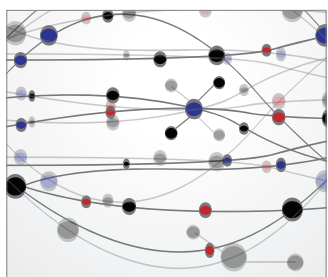

The Scientific World Journal
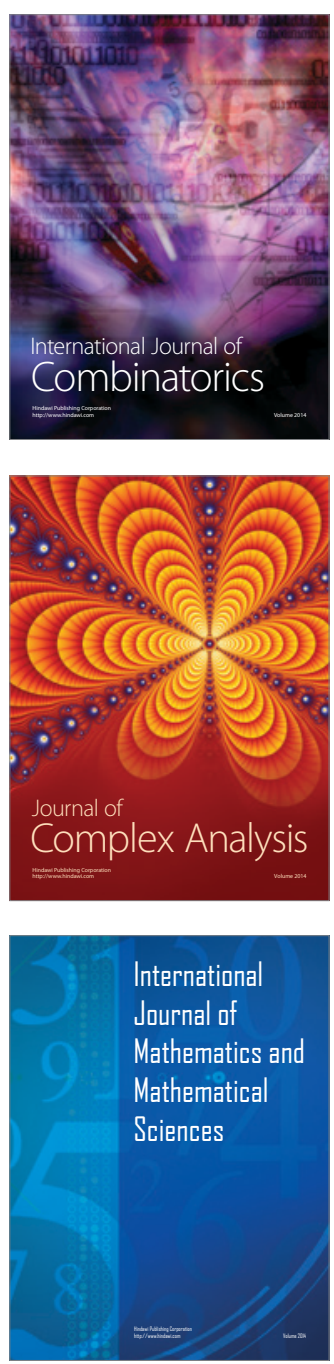
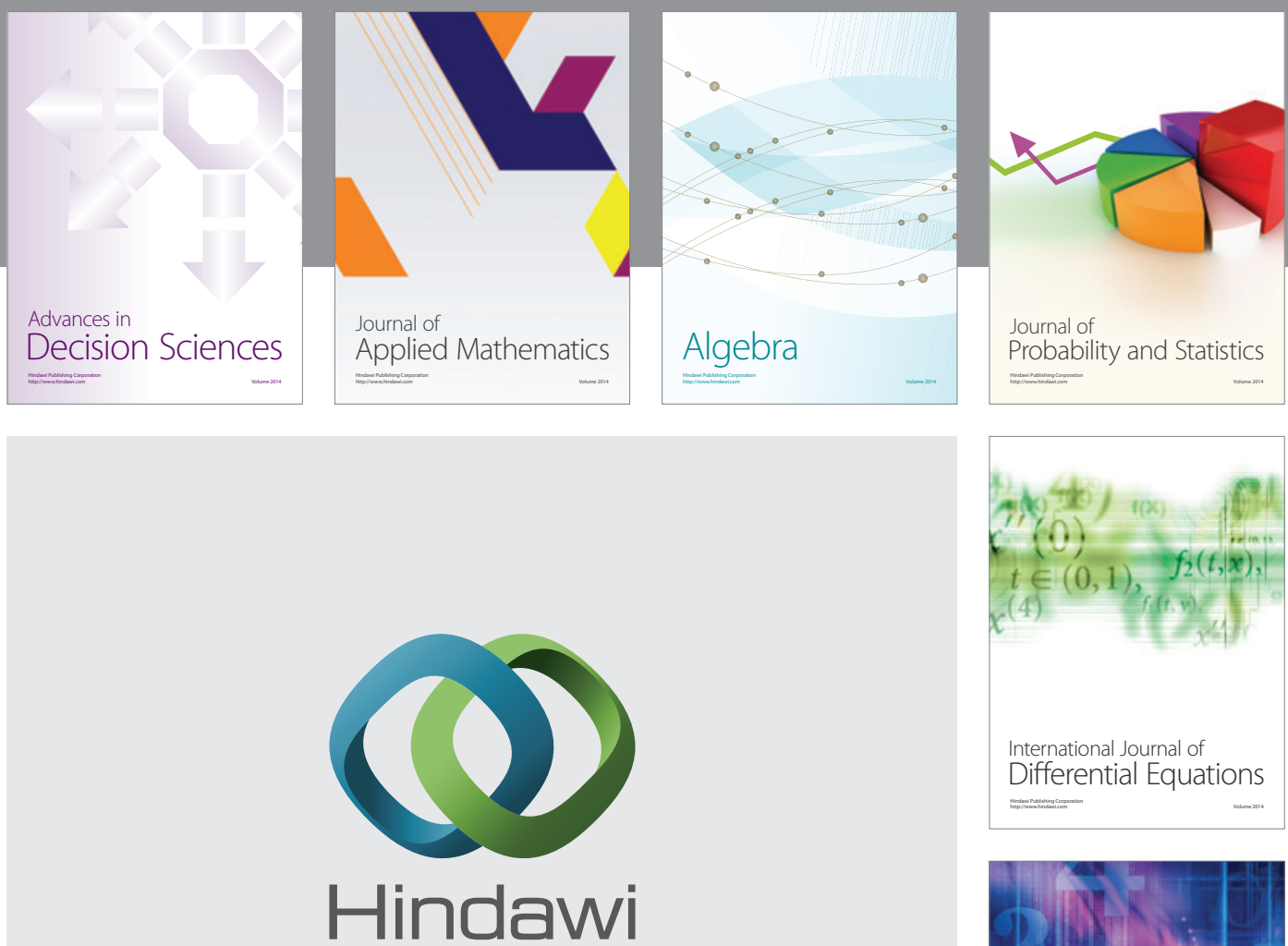

Submit your manuscripts at http://www.hindawi.com
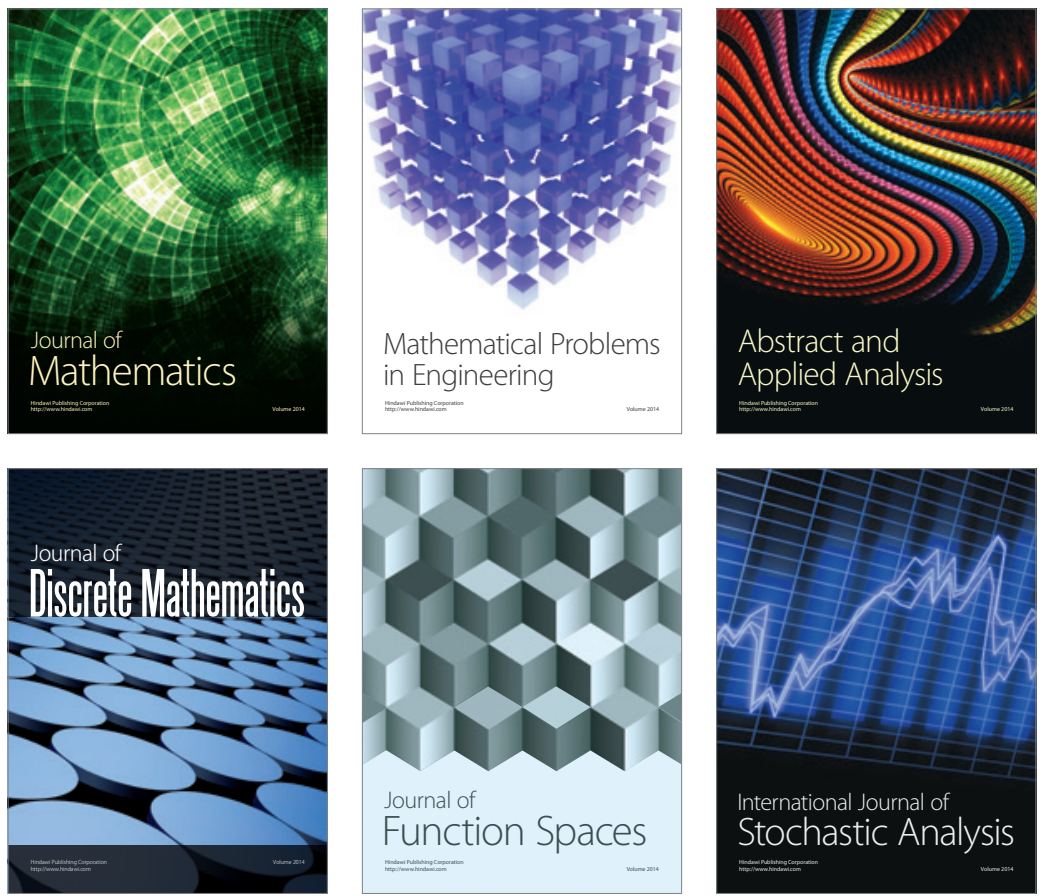

Journal of

Function Spaces

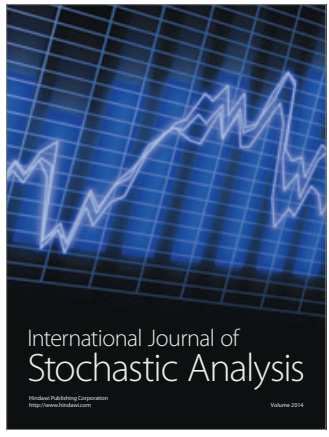

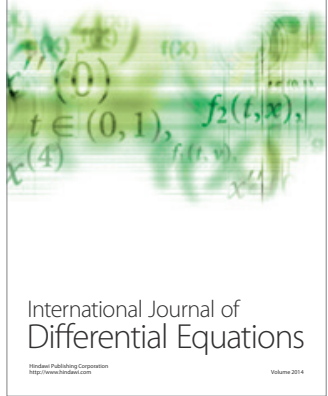
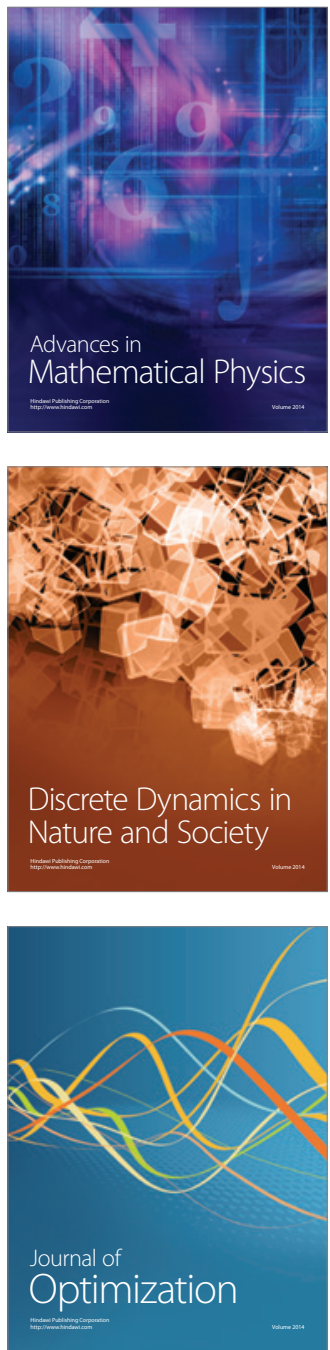